Supplementary Information

\title{
Transition Metal-Free Half-Metallicity in Two-Dimensional Gallium Nitride with Quasi-Flat Band
}

Seungjun Lee, ${ }^{\dagger, \ddagger}$ Hussain Alsalman, ${ }^{\ddagger}$ Wei Jiang, ${ }^{\ddagger}$ Tony Low, ${ }^{\dagger, \ddagger}, \boldsymbol{\Phi}$ and Young-Kyun Kwon*,†,§

$\dagger$ Department of Physics, Kyung Hee University, Seoul, 02447, Korea $\ddagger$ Department of Electrical and Computer Engineering, University of Minnesota, Minneapolis, Minnesota 55455, USA

9 Department of Physics, University of Minnesota, Minneapolis, Minnesota 55455, USA $\S$ Department of Information Display and Research Institute for Basic Sciences, Kyung Hee University, Seoul, 02447, Korea

E-mail: ykkwon@khu.ac.kr 


\section{S1 Computational details}

To investigate the electronic structures of various configurations including a 2D-GaN structure, we first constructed their corresponding model structures in a hexagonal lattice. The equilibrium lattice constant of a freestanding 2D-GaN was calculated to be $3.216 \AA$, and the lattice constant of 2D-GaN|SiC(0001) and 2D-GaN|w-GaN(0001) were chosen to be $3.098 \AA$ and $3.242 \AA$, respectively, which are equilibrium lattice constants of bulk SiC and w-GaN substrates determined by DFT calculation. For 2D-GaN on a substrate, either SiC(0001) or w-GaN(0001), with a graphene overlayer, we constructed a $4 \times 4 \times 1$ supercell structure of 2D-GaN on the substrate with a $5 \times 5 \times 1$ cell structure of graphene to minimize their lattice mismatch. Graphene overlayer undergoes an artificial tensile strain of $1.25 \%$ induced by lattice mismatch with the $\mathrm{SiC}$ substrates.

The electronic wavefunctions were expanded by planewave basis with kinetic energy cutoff of $500 \mathrm{eV}$. We employed the projector-augmented wave pseudopotentials ${ }^{\mathrm{S} 1, \mathrm{~S} 2}$ to describe the valence electrons, and treated We employed Grimme-D2 Van der Waals correction ${ }^{\mathrm{S} 3}$ to describe long range interaction between graphene and 2D-GaN, and included a sufficiently large vacuum region to mimic $2 \mathrm{D}$ layered or slab structure in periodic cells. The corresponding Brillouin zone was sampled using a $30 \times 30 \times 1 k$-point mesh according to the Monkhost-Pack scheme ${ }^{\mathrm{S} 4}$ for the primitive unit cell of $2 \mathrm{D}-\mathrm{GaN}$, and a a $4 \times 4 \times 1 k$-grid for the supercell structures.

To explore finite size effects, we employed $L \times L$ supercells with $L=4 \sim 80$, and performed our MC simulations at various temperatures. We carried out MC simulation over $2 \times 10^{4} \times L^{2}$ steps for each supercell with $L$, and collected data after the system reached its equilibrium to extract the magnetic properties. 
(a)
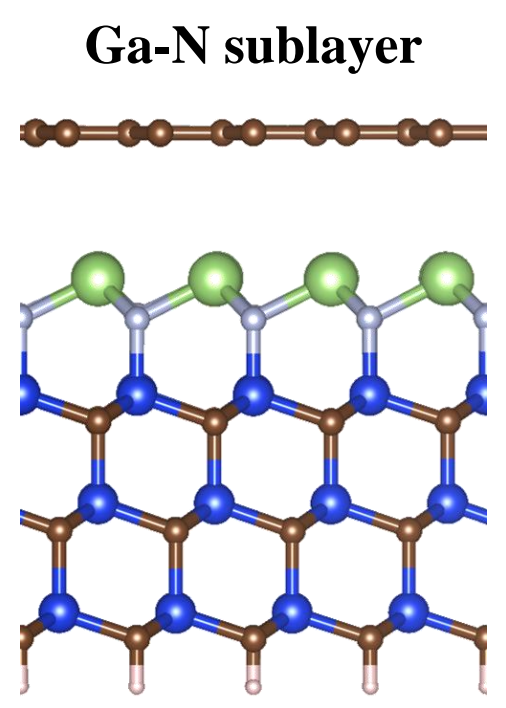

(b) $\quad \mathrm{N}$-Ga sublayer
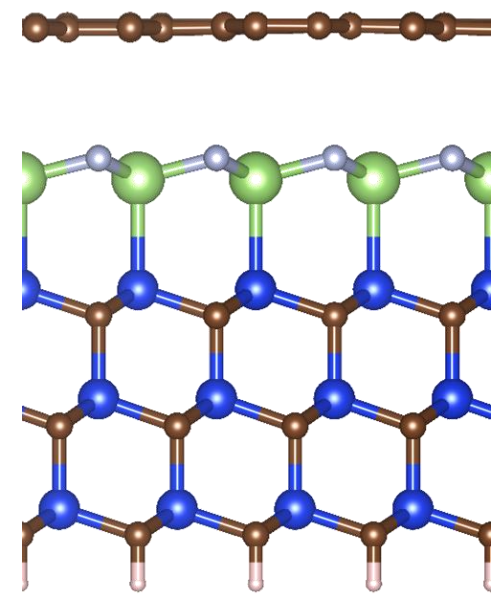

Figure S1: Heterostructure configurations of graphene-encapsulated (a) Ga-N sublayer and (b) N-Ga sublayer on Si-polar $\mathrm{SiC}(0001)$. The dangling bonds at the bottom of $\mathrm{SiC}$ substrate are saturated by hydrogen atoms. Brown, grey, green, blue, and pink spheres indicate carbon, nitrogen, gallium, silicon, and hydrogen atoms, respectively. 


\section{S2 Initial growth stage of the 2D-GaN}

At an initial growth stage of the $2 \mathrm{D} \mathrm{GaN}$ on the $\mathrm{SiC}$ substrate, there are two possible growth pathways, one is forming a (Ga-polar) Ga-N sublayer and the other a (N-polar) NGa sublayer. To compare their relative stability, we calculate total energies of both the Ga-N and the N-Ga sublayer on a $\mathrm{Si}$-polar $\mathrm{SiC}(0001)$ substrate either with or without graphene layer, as shown in Fig. S1. The total energies of the $\mathrm{Ga}-\mathrm{N} \mid \mathrm{SiC}$ and $\mathrm{N}-\mathrm{Ga} \mid \mathrm{SiC}$ were calculated to be -59.92 and $-58.62 \mathrm{eV}$, or -1453.18 and $-1432.56 \mathrm{eV}$ either without or with the top graphene layer, respectively. Therefore, regardless of whether the graphene covers or not, the Ga-N sublayer is preferred to grow on the $\mathrm{SiC}$ substrate. 

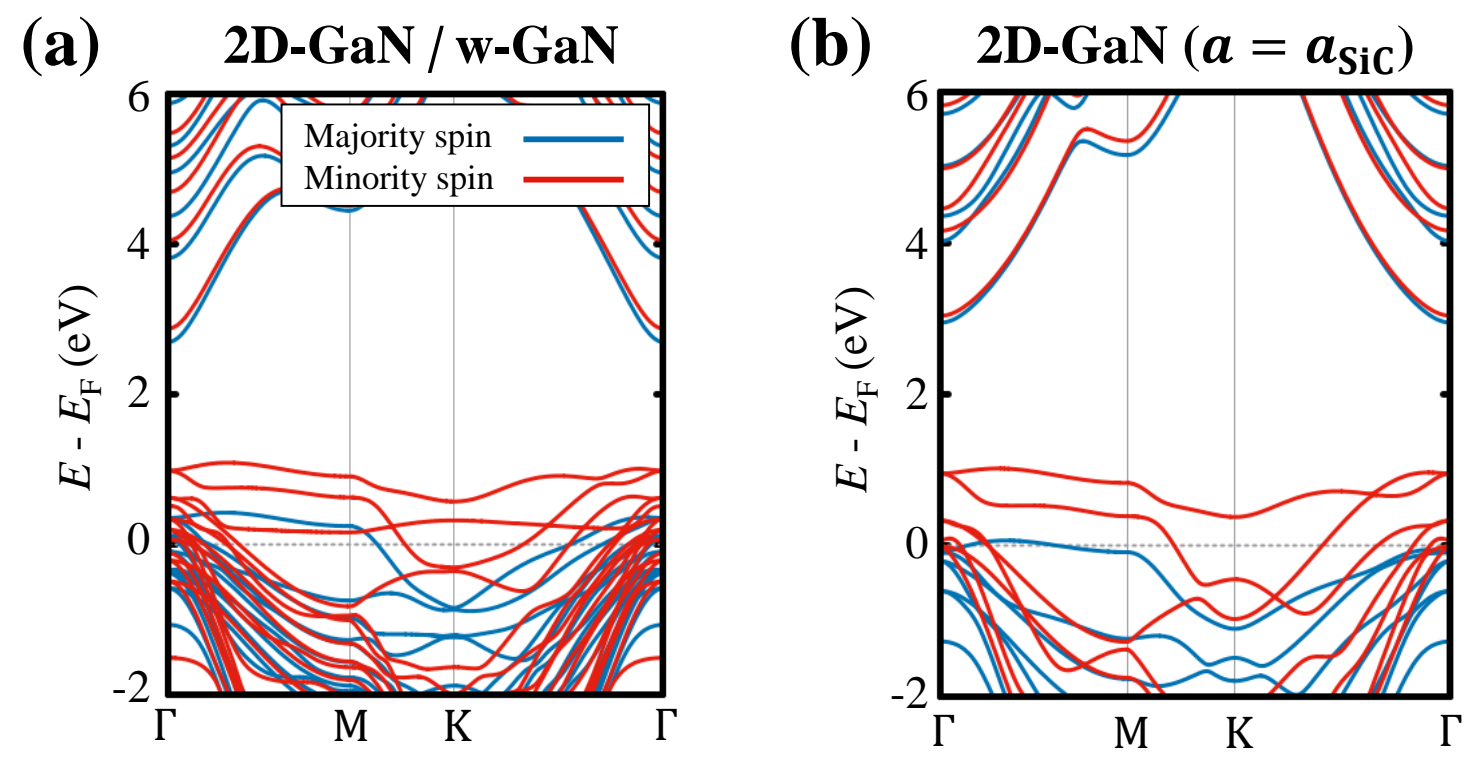

Figure S2: Spin-polarized electronic structure of (a) 2D-GaN|Ga-polar w-GaN(0001) and (b) freestanding 2D-GaN with lattice constant of $\mathrm{SiC}$ substrate $a_{\mathrm{SiC}}$. Red and blue solid lines indicate minority and majority spin states. 

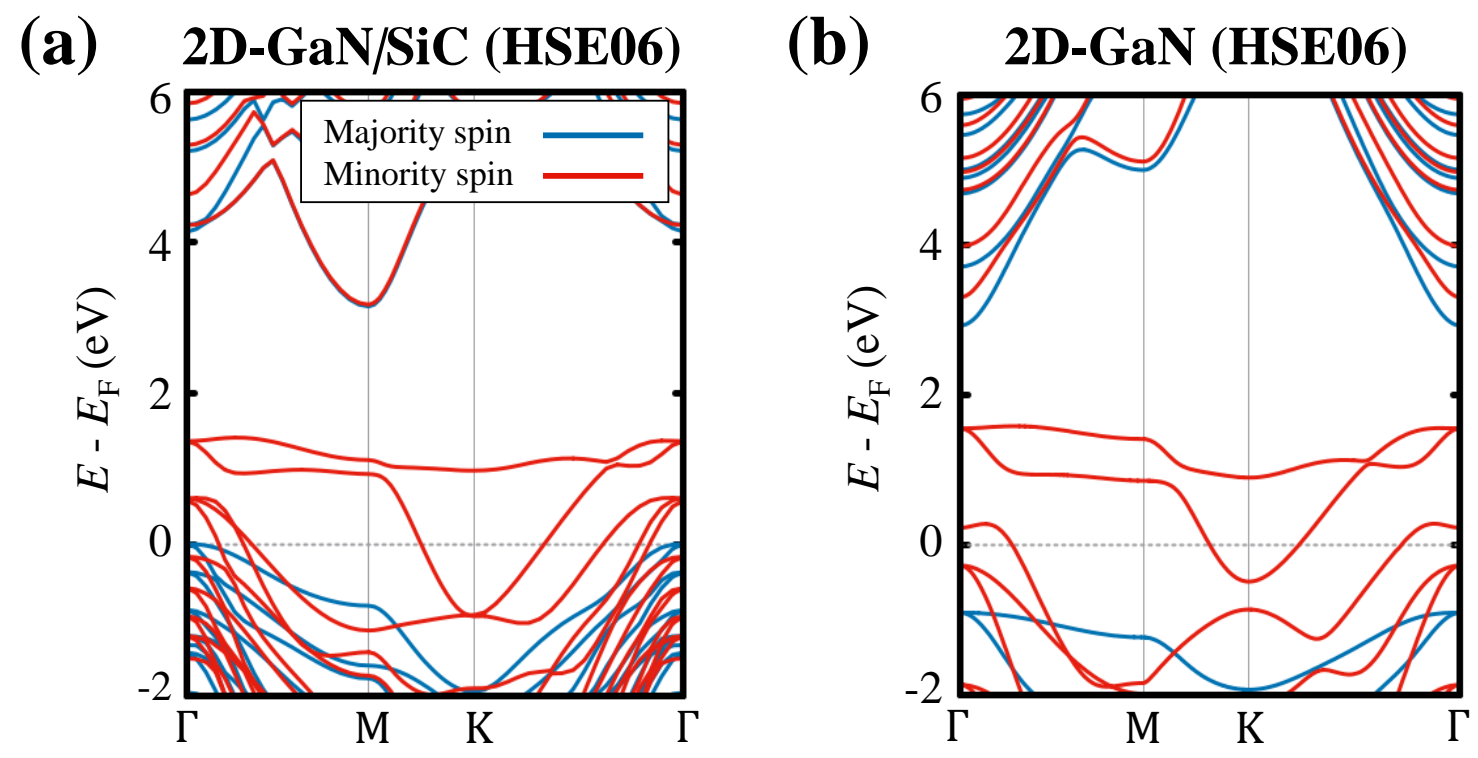

Figure S3: Spin-polarized electronic structure calculated by HSE06 XC functional for (a) $2 \mathrm{D}-\mathrm{GaN} \mid \mathrm{SiC}$, and (b) free-standing $2 \mathrm{D}-\mathrm{GaN}$ with a lattice constant of SiC substrate $a_{\mathrm{SiC}}$. Red and blue solid lines indicate minority and majority spin states. 


\section{S3 Dependence of exchange-correlation functionals on low- dimensional itinerant magnet}

For magnetic insulators, in general, the magnetic order is mainly induced by the strong correlation effect of localized electrons. Thus, the PBE functional often fails to describe such localized magnetism. Instead, high level methods such as hybrid functional, $\mathrm{PBE}+U$ or LDA $+U$ are usually used for those localized magnetic systems to correctly describe the correlation effects. For conventional itinerant magnetic systems such as bulk Fe, Co, and $\mathrm{Ni}$, on the contrary, it has been reported that the PBE functional yields more accurate magnetic moment than the hybrid functional, which overestimates their exchange splitting due to the strong screening in metal. ${ }^{\mathrm{S5}, \mathrm{S6}}$ On the other hand, $2 \mathrm{D}-\mathrm{GaN}$ cannot be classified exclusively into either one of magnetic insulator or itinerant magnet, since it is a half metallic ferromagnet with nearly localized electrons due to quasi-flat band. Thus, 2D-GaN can be regarded as an intermediate system exhibiting both localized and itinerant magnetic characteristics and the actual value of its magnetic moment would fall in between those values evaluated by the PBE and HSE06 funcionnals. For more accurate and quantitative analysis, we are waiting for experimental observations on the magnetic property of 2D-GaN. At this very moment, therefore, we believe that the PBE functional is regarded as a safe choice avoiding the possibility of overestimation. 


\section{S4 Comparison between 2D-GaN and other reported 2D $p$-half-metallic materials}

It is worth noting that the half-metallicity of $2 \mathrm{D}-\mathrm{GaN}$ is superior to that of other transition-metal-free $p$-half-metallic materials. As described the main manuscript, halfmetallic characteristic of 2D-GaN would emerge under a weak magnetic field, but without any carrier doping or applied external electric field. More importantly, this material has been experimentally produced. ${ }^{\mathrm{S} 14}$ In Table S1, we compared the spin splitting energy and Curie temperature of the 2D-GaN with those available for other reported 2D p-half-metallic materials. Since there has been no estimation of $T_{\mathrm{C}}$ based on a Heisenberg model Hamiltonian with SIMAE and external field effect, our comparison can be only possible for the estimation based on DFT-based MFA or Ising model, by both of which the Curie temperature was overestimated. Nevertheless, this relative comparison would provide a hint for better materials. Similar to our freestanding $2 \mathrm{D}-\mathrm{GaN}, \mathrm{g}-\mathrm{C}_{4} \mathrm{~N}_{3}$ does not require any carrier doping or applied external electric field. ${ }^{\mathrm{S} 7}$ On the other hand, there are some other $2 \mathrm{D}$ layered semiconductors, such as GaSe, $\mathrm{C}_{2} \mathrm{~N}$, phosphorene, arsenene, and NSb, undergoing the half-metallic phase transition with a strong carrier doping, which leads to the Stoner instability by shifting the Fermi levels toward the states exhibiting the van Hoff singularity originating from the low dimensionality. ${ }^{\text {S8-S11 }}$ In many cases, however, the amount of hole doping required for such transition is too high to be practical in real experiments. It was, for example, reported that the required hole doping amount for NSb to be half-metallic with $T_{\mathrm{C}}^{\text {Ising }}=617 \mathrm{~K}$ is approximately 0.6 carriers per unit cell, ${ }^{\mathrm{S} 11}$ indicating that it would be nearly impossible to achieve in reality. We found, on the contrary, that a small amount of electron doping can restore the half metallicty of $2 \mathrm{D}-\mathrm{GaN}$ grown on $\mathrm{SiC}$ substrate that lost its half metallicity due to its smaller lattice constant than its freestanding value, as indicated by its band structure shown in Fig. 2(b). The estimated doping amount is $\lesssim 0.1 e /$ cell. It was also 
reported that another group of $2 \mathrm{D}$ materials comprising transition-metal dinitrides exhibits intrinsic half-metallicity, which intriguingly originates from the $p$-orbitals of nitrogen atoms rather than the $d$-orbitals of transition-metal atoms. ${ }^{\mathrm{S} 12, \mathrm{~S} 13}$ As summarized in Table S1, our proposed 2D-GaN would be a relatively better $p$-half-metallic material than these candidate materials. It can be easily grown on any hexagonal substrates available in the current semiconductor industry. By selecting an adequate substrate or by adding a small amount of electrons, it can restore its half metallicity with high magnetic moment, which are the intrinsic characteristic of its freestanding counterpart. Moreover, it exhibits highest spin splitting energy of $\Delta \sim 1.18 \mathrm{eV}$ and the Curie temperature of $T_{\mathrm{C}} \approx 165 \mathrm{~K}$. 
Table S1: The computationally evaluated spin splitting energies $(\Delta)$ and Curie temperatures of various 2D $p$-orbital-based half-metallic materials to compare with those of our system, 2D-GaN $\left(a=1.00 a_{\mathrm{SiC}}\right)$. These materials were classified into three categories: (i) transition-metal-free intrinsic half-metals, (ii) transition-metal-free half-metals induced by hole doping, and (iii) half-metallic transition metal dinitrides. Listed are three kinds of Curie temperatures, $T_{\mathrm{C}}^{\mathrm{MFA}}$, $T_{\mathrm{C}}^{\text {Ising }}$, and $T_{\mathrm{C}}^{\text {Heisen }}$ estimated by different schemes, DFT-based mean field approximation, Ising model, and Heisenberg model with a weak external $B$ field, respectively.

\begin{tabular}{ccccccc}
\hline \hline Categories & Materials & $\Delta(\mathrm{eV})$ & $T_{\mathrm{C}}^{\mathrm{MFA}}(\mathrm{K})$ & $T_{\mathrm{C}}^{\text {Ising }}(\mathrm{K})$ & $\begin{array}{c}T_{\mathrm{C}}^{\text {Heisen }}(\mathrm{K}) \\
(\mathrm{w} / \text { weak } B)\end{array}$ & References \\
\hline \multirow{2}{*}{ (i) } & $2 \mathrm{D}-\mathrm{GaN}$ & $\sim 1.18$ & 4000 & 522 & $\sim 165$ & This work \\
& $\mathrm{g}-\mathrm{C}_{4} \mathrm{~N}_{3}$ & $\sim 0.8$ & & & & $\mathrm{~S} 7$ \\
\hline \multirow{4}{*}{ (ii) } & $\mathrm{GaSe}$ & $\sim 0.06$ & & 91 & & $\mathrm{~S} 8$ \\
& $\mathrm{C}_{2} \mathrm{~N}$ & $\sim 0.1$ & 320 & & & $\mathrm{~S} 9$ \\
& Phosphorene & $\sim 0.5$ & 1400 & & & $\mathrm{~S} 10$ \\
& Arsenene & $\sim 0.29$ & 820 & & $\mathrm{~S} 10$ \\
& $\mathrm{NSb}$ & $\sim 1.2$ & 2600 & 617 & & $\mathrm{~S} 11$ \\
\hline \multirow{2}{*}{ (iii) } & $1 \mathrm{H}-\mathrm{MoN}_{2}$ & $\sim 1.0$ & & 420 & $\mathrm{~S} 12$ \\
& $1 \mathrm{~T}-\mathrm{TaN}_{2}$ & $\sim 1.0$ & 556 & & $\mathrm{~S} 13$ \\
\hline \hline
\end{tabular}



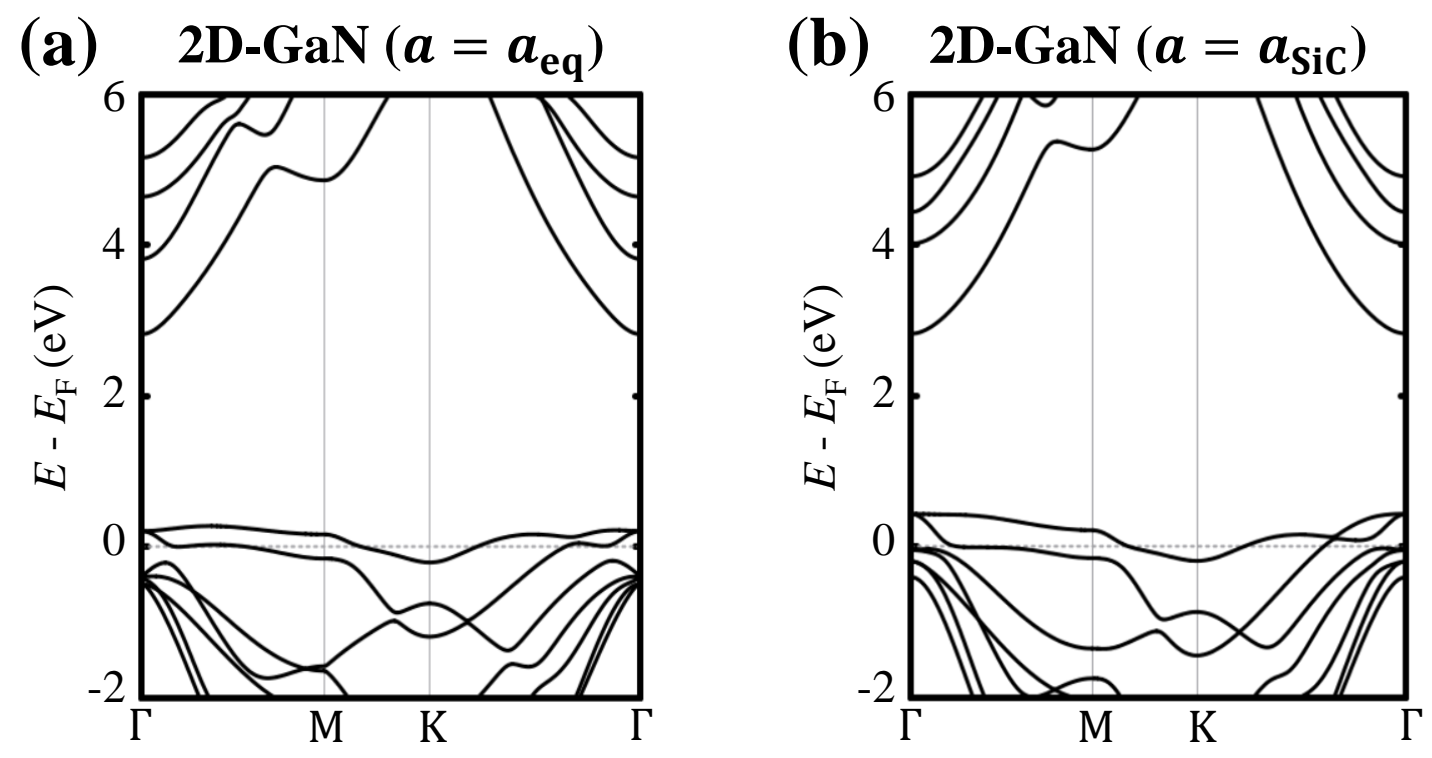

Figure S4: Non-magnetic electronic band structures of free-standing 2D-GaN with lattice constant (a) $a=a_{\mathrm{eq}}$ of its equilibrium structure and (b) $a=a_{\mathrm{SiC}}$ of $\mathrm{SiC}$ substrate. The former structure exhibits even flatter near the Fermi level than the latter. 

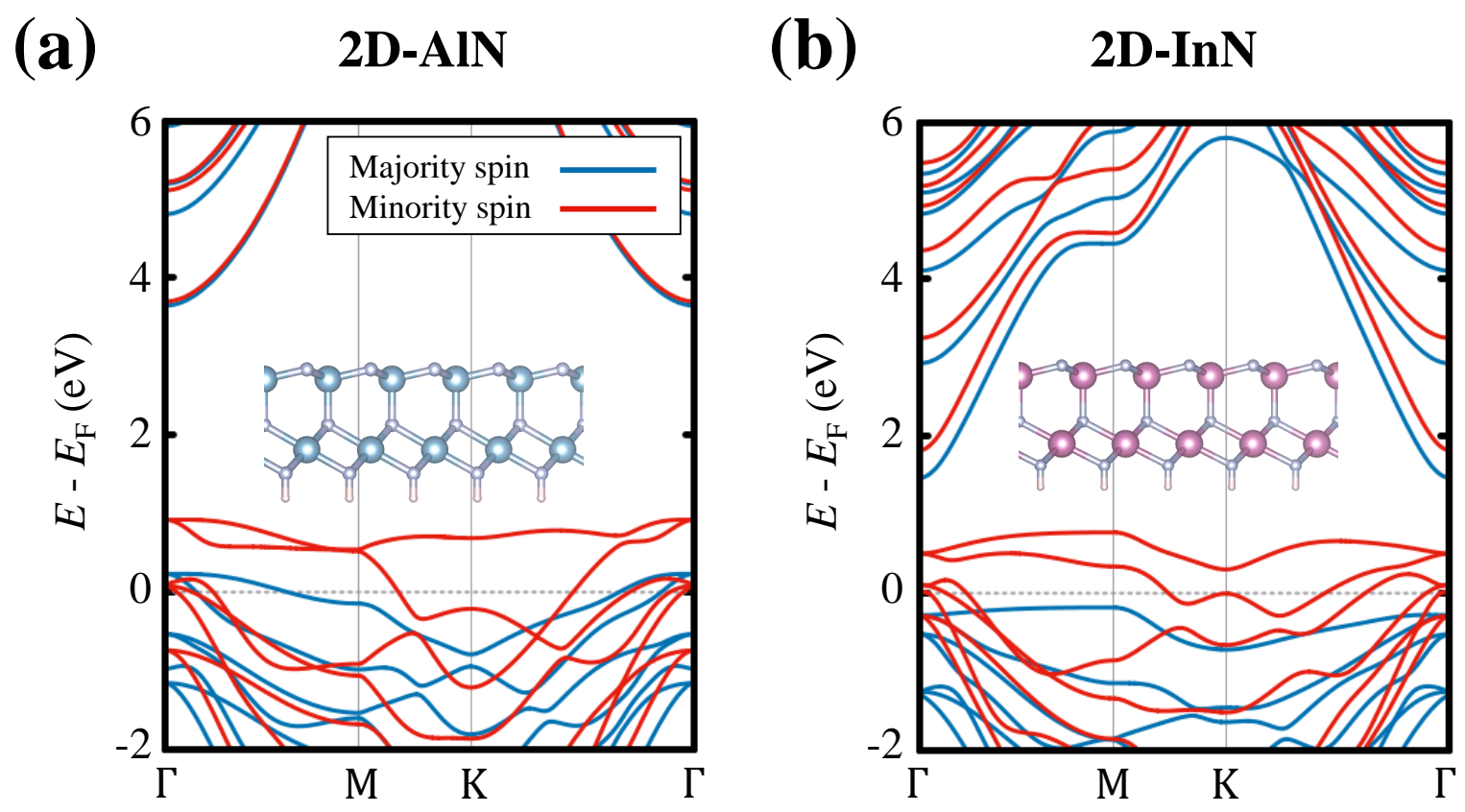

Figure S5: Spin-polarized electronic structure of (a) free-standing 2D-AlN and (b) 2D-InN respectively. Red and blue solid lines indicate minority and majority spin states. Their crystal structures in which $\mathrm{N}, \mathrm{Al}$, and In atoms are depicted by the grey, blue, and purple spheres, are overlaid in (a) and (b). The dangling bond of each $\mathrm{N}$ atom in both structures is saturated with a hydrogen atom depicted with the pink sphere. 


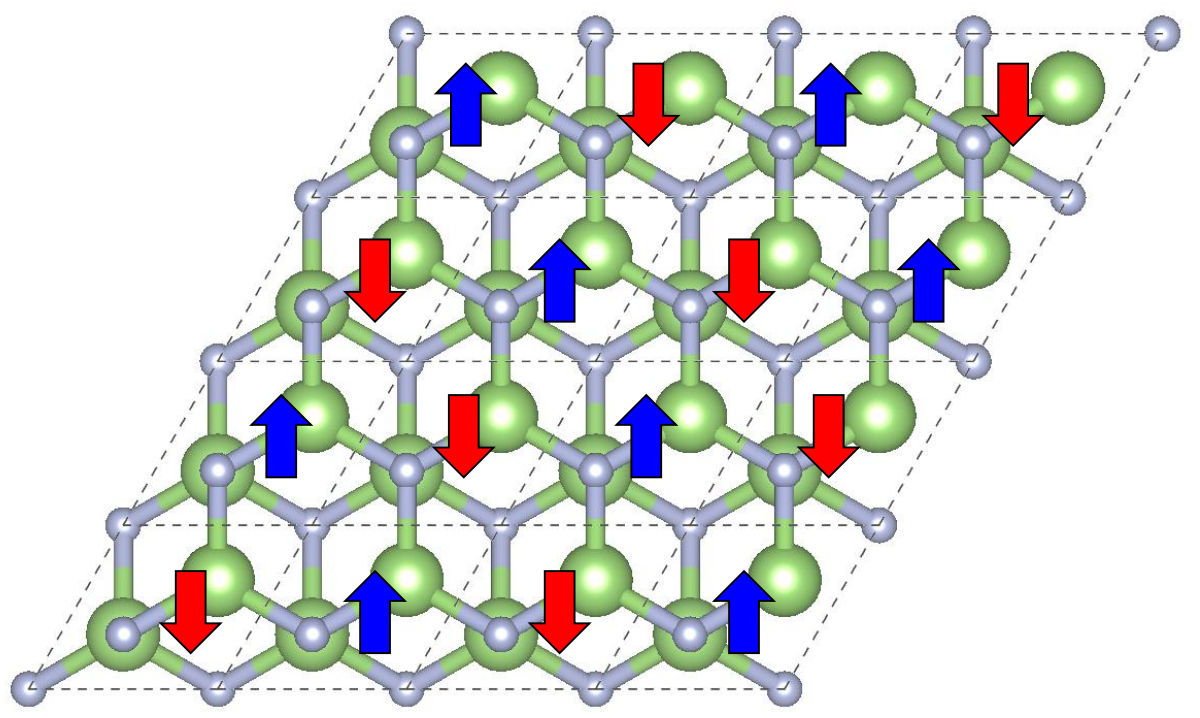

Figure S6: Anti-ferromganetic configuration of 2D-GaN. Grey and green spheres indicate nitrogen and gallium atoms, and red and blue arrows indicate up and down spin state. Dashed line shows the primitive unit cell. Each nitrogen in a single unit cell has same spin configuration. 


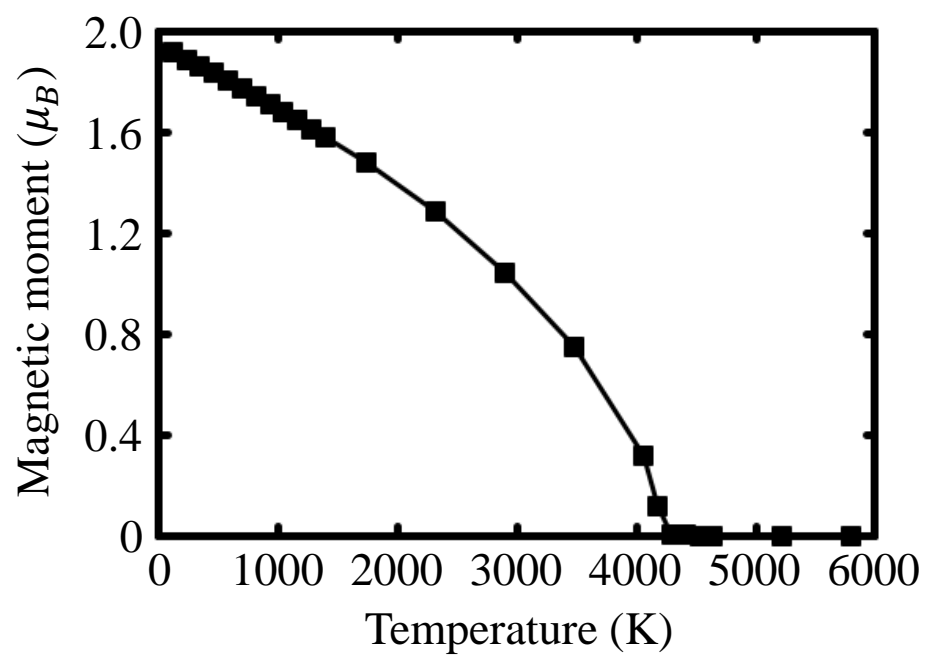

Figure S7: Temperature dependant magnetic moment estimated by DFT-based mean-field approximation (MFA). The MFA estimate unrealistic Curie temperature $\left(T_{\mathrm{C}}^{\mathrm{MFA}} \gtrsim 4000 \mathrm{~K}\right)$, since it improperly describes low-energy excitation. ${ }^{\mathrm{S} 11, \mathrm{~S} 15}$ 
(a)

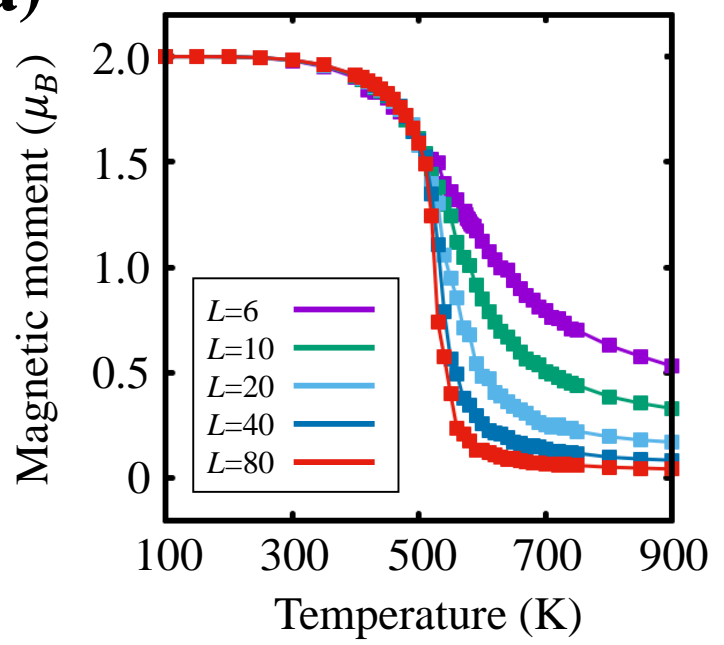

(c)

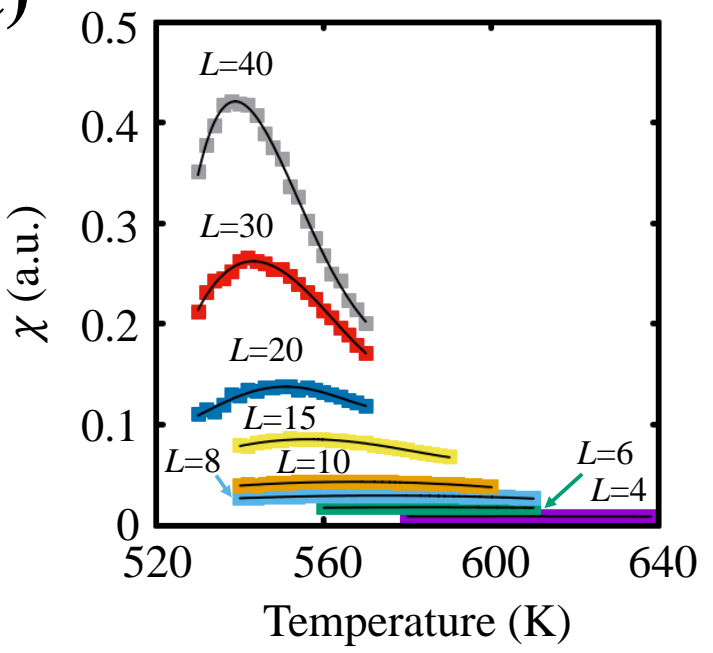

(b)

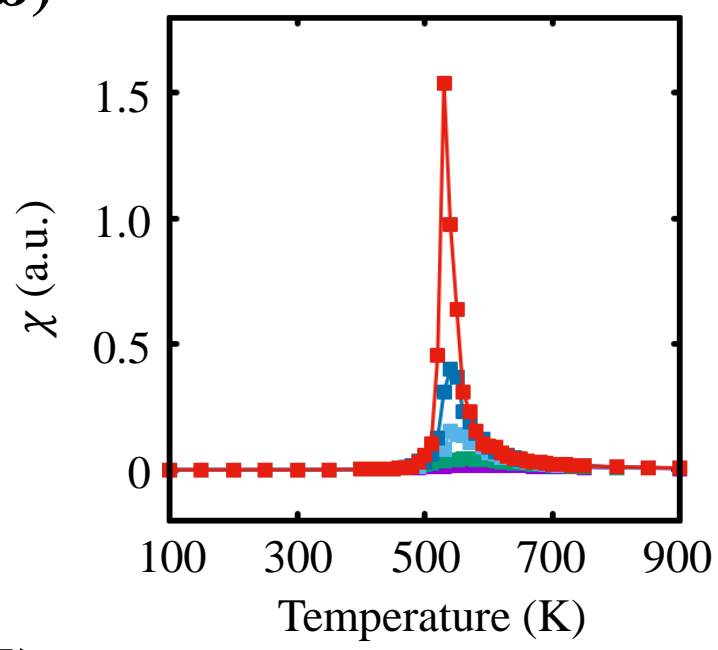

(d)

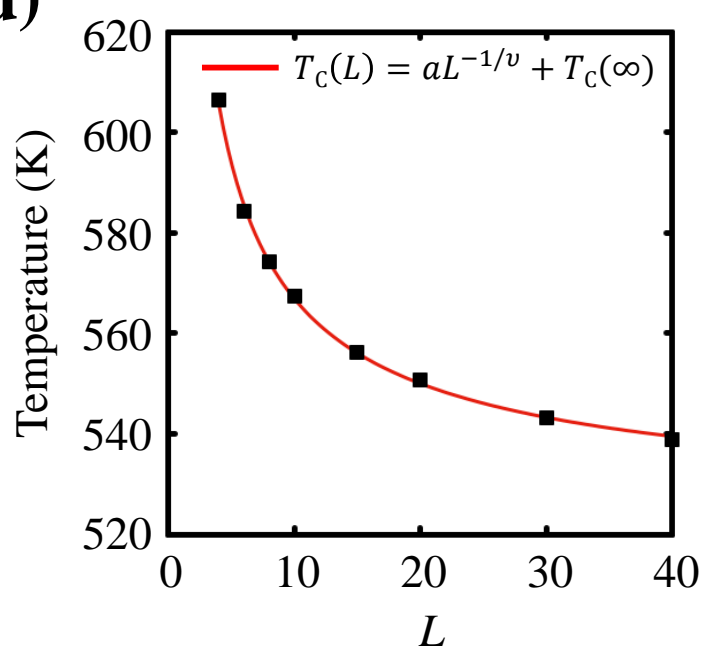

Figure S8: Temperature-dependant (a) magnetic moment and (b) magnetic susceptibility $\chi$ of the 2D-GaN evaluated by taking ensemble averages from Monte Carlo simulation based on Ising model Hamiltonian performed in different-sized supercells with $L \times L(L=6,10,20,40$ and 80). (c) Temperature dependant magnetic susceptibility $\chi$ of 2 D-GaN nearby transition temperature for different $L \times L$ supercells. The black solid line in each case indicates a corresponding polynomial fitting function. The each point nearby transition temperature was carefully evaluated by using sufficiently long MC simulation over $L^{2} \times 10^{6}$ steps. (d) Finite size scaling of the Curie temperature of 2D-GaN. Each black dot represents the Curie temperature evaluated at the maximum value of $\chi$ for each given supercell size, which were fitted with the fitting function $T_{\mathrm{C}}(L)=a L^{-1 / \nu}+T_{\mathrm{C}}$ plotted in the red solid line. This finite size fitting procedure yielded the Curie temperature $T_{\mathrm{C}}$ to be $522 \mathrm{~K}$ with the other fitting parameters $a$ and $\nu$ to be $216 \mathrm{~K}$ and 1.46 , respectively. 

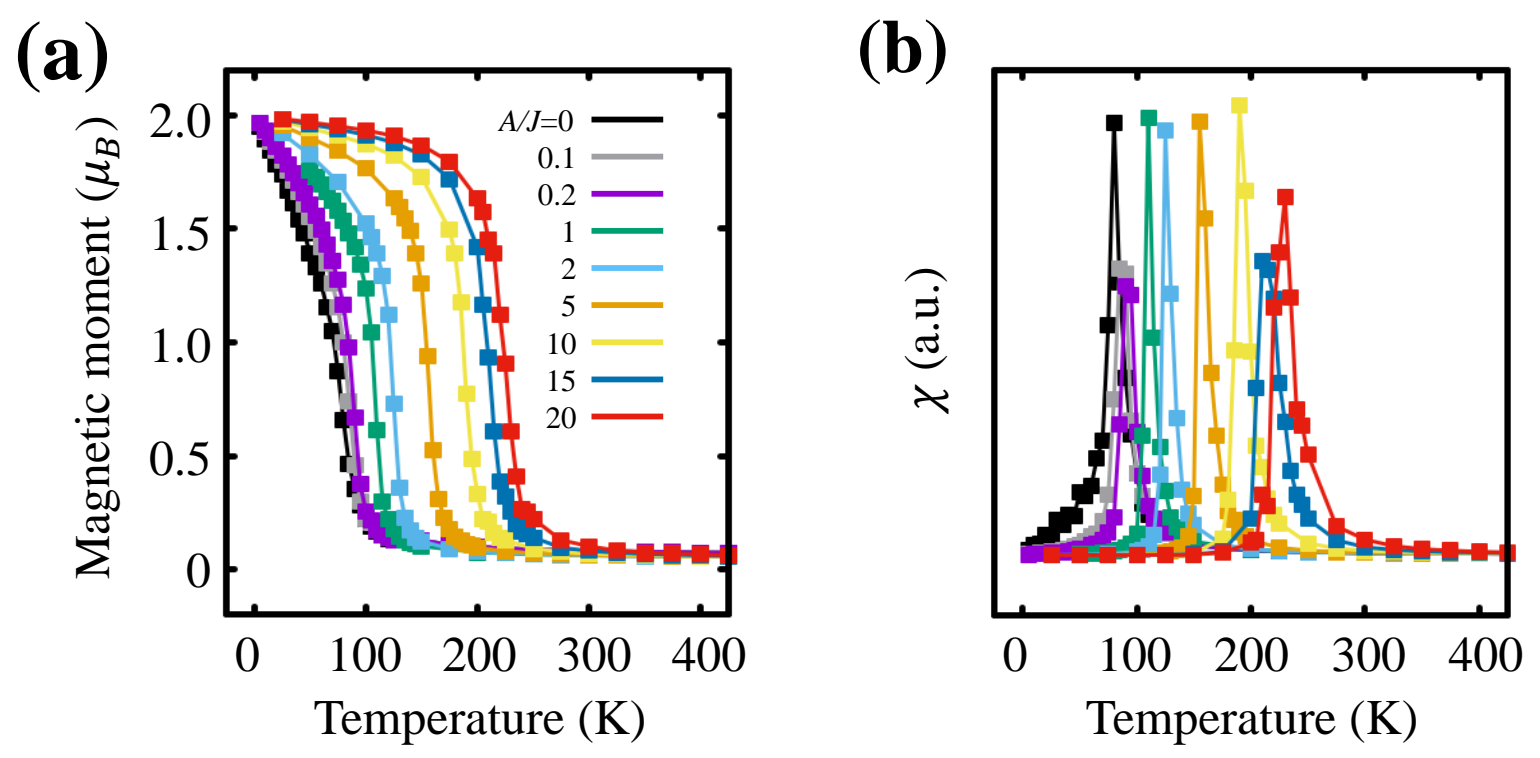

Figure S9: Temperature-dependant (a) magnetic moment and (b) magnetic susceptibility $\chi$ of 2D-GaN evaluated by taking ensemble averages from Monte Carlo simulation based on Heisenberg model Hamiltonian defined as Eq. (1) in the main manuscript. The simulation was performed with different values of single ion anistropy $A$ while keeping a magnetic field $B$ as zero. The $40 \times 40$ supercell was used in this simulation. 

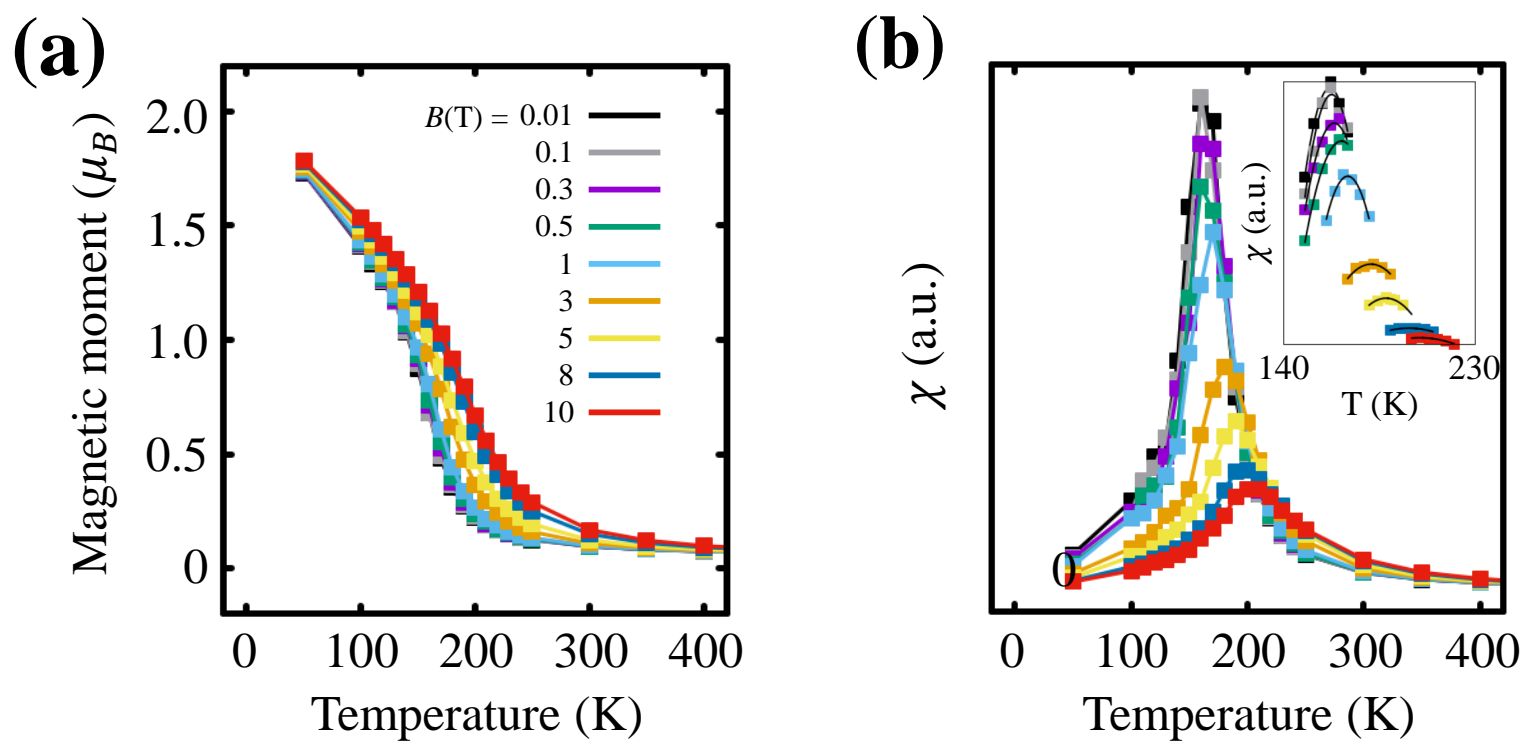

Figure S10: Temperature-dependant (a) magnetic moment and (b) magnetic susceptibility $\chi$ of 2D-GaN evaluated by taking ensemble averages from Monte Carlo simulation based on Heisenberg model Hamiltonian defined as Eq. (1) in the main manuscript. The simulation was performed with different values of applied external magnetic field $B$ while keeping single ion anistropy $A$ as zero. Inset in (b) shows a close-up view of the magnetic susceptibility $\chi$ of $2 \mathrm{D}-\mathrm{GaN}$ nearby the transition temperature with varying external magnetic field. The black solid line in each case indicates a corresponding polynomial fitting function. The $40 \times 40$ supercell was used in this simulation. 


\section{References}

[S1] Blöchl, P. E. Projector Augmented-Wave Method. Phys. Rev. B 1994, 50, 1795317979.

[S2] Kresse, G.; Joubert, D. From Ultrasoft Pseudopotentials to the Projector AugmentedWave Method. Phys. Rev. B 1999, 59, 1758-1775.

[S3] Grimme, S. Semiempirical GGA-Type Density Functional Constructed with a LongRange Dispersion Correction. J. Comput. Chem. 2006, 27, 1787-1799.

[S4] Monkhorst, H. J.; Pack, J. D. Special Points for Brillouin-Zone Integrations. Phys. Rev. B 1976, 13, 5188.

[S5] Gao, W.; Abtew, T. A.; Cai, T.; Sun, Y.-Y.; Zhang, S.; Zhang, P. On the Applicability of Hybrid Functionals for Predicting Fundamental Properties of Metals. Solid State Commun. 2016, 234-235, 10-13.

[S6] Paier, J.; Marsman, M.; Hummer, K.; Kresse, G.; Gerber, I. C.; Ángyán, J. G. Screened Hybrid Density Functionals Applied to Solids. J. Chem. Phys. 2006, 124, 154709.

[S7] Du, A.; Sanvito, S.; Smith, S. C. First-Principles Prediction of Metal-Free Magnetism and Intrinsic Half-Metallicity in Graphitic Carbon Nitride. Phys. Rev. Lett. 2012, 108, 197207.

[S8] Cao, T.; Li, Z.; Louie, S. G. Tunable Magnetism and Half-Metallicity in Hole-Doped Monolayer GaSe. Phys. Rev. Lett. 2015, 114, 236602.

[S9] Gong, S.; Wan, W.; Guan, S.; Tai, B.; Liu, C.; Fu, B.; Yang, S. A.; Yao, Y. Tunable Half-Metallic Magnetism in an Atom-Thin Holey Two-Dimensional $\mathrm{C}_{2} \mathrm{~N}$ Monolayer. $J$. Mater. Chem. C 2017, 5, 8424-8430.

[S10] Fu, B.; Feng, W.; Zhou, X.; Yao, Y. Effects of Hole Doping and Strain on Magnetism in Buckled Phosphorene and Arsenene. 2D Mater. 2017, 4, 025107. 
[S11] Nie, Y.; Rahman, M.; Liu, P.; Sidike, A.; Xia, Q.; Guo, G.-h. Room-Temperature Half-Metallicity in Monolayer Honeycomb Structures of Group-V Binary Compounds with Carrier Doping. Phys. Rev. B 2017, 96, 075401.

[S12] Wu, F.; Huang, C.; Wu, H.; Lee, C.; Deng, K.; Kan, E.; Jena, P. Atomically Thin Transition-Metal Dinitrides: High-Temperature Ferromagnetism and Half-Metallicity. Nano Lett. 2015, 15, 8277-8281.

[S13] Liu, J.; Liu, Z.; Song, T.; Cui, X. Computational Search for Two-Dimensional Intrinsic Half-Metals in Transition-Metal Dinitrides. J. Mater. Chem. C 2017, 5, 727-732.

[S14] Al Balushi, Z. Y.; Wang, K.; Ghosh, R. K.; Vilá, R. A.; Eichfeld, S. M.; Caldwell, J. D.; Qin, X.; Lin, Y.-C.; DeSario, P. A.; Stone, G. et al. Two-Dimensional Gallium Nitride Realized via Graphene Encapsulation. Nature Mater. 2016, 15, 1166-1171.

[S15] Kübler, J. Theory of Itinerant Electron Magnetism; Clarendon Press, Oxford, 2000. 